\title{
Soil Moisture by Remote Sensing Retrieval in the Tropic of Cancer of Yunnan Province
}

\author{
Yuanhe $\mathbf{Y u}^{1-3}$, Jinliang Wang ${ }^{1-3 *}$, Feng Cheng ${ }^{1-3}$, Yun Chen ${ }^{1-3}$
}

${ }^{1}$ College of Tourism and Geographic Sciences, Yunnan Normal University, Kunming, Yunnan, China ${ }^{2}$ Key Laboratory of Resources and Environmental Remote Sensing for Universities in Yunnan, Kunming, Yunnan, China ${ }^{3}$ Center for Geospatial Information Engineering and Technology of Yunnan Province, Kunming, Yunnan, China

Received: 3 May 2019

Accepted: 23 June 2019

\begin{abstract}
Soil moisture is a direct source of water for terrestrial plants. Measurement of soil moisture can help to characterize plant growth and portray regional soil moisture information in a timely manner, which is of great significance for water and soil regulation. This study uses the Tropic of Cancer in Yunnan Province as a research area, and based on MODIS product data, the apparent thermal inertia (ATI), vegetation supply water index (VSWI) and surface water capacity index (SWCI) are calculated. The measured data are used in regression analysis modeling to retrieve the soil moisture in the study area. The results show that the three indices of ATI, SWCI and VSWI have good correlation with soil moisture, among which VSWI has the best fitting effect, and ATI and SWCI can retrieve soil moisture more effectively. The soil moisture contents are mostly concentrated in the range of 0.2 to $0.4 \mathrm{~m}^{3} / \mathrm{m}^{3}$, among which the soil moisture of Jianshui County, Mengzi City, Wenshan County, Yanshan County, Gejiu City and Shiping County are the smallest, and the soil moisture in the western portion of Gengma County and Cangyuan County and the central portion of Jinggu County are the largest. As the fractional vegetation cover increases, the soil moisture content also gradually increases, and the mean value is greater than $0.2 \mathrm{~m}^{3} / \mathrm{m}^{3}$.
\end{abstract}

Keywords: MODIS data, soil moisture, remote sensing retrieval, Tropic of Cancer

\section{Introduction}

Soil moisture (SM) is an important indicator of climate and environmental drought, the basis of sustainable and healthy operation of global ecosystems [1], and an important parameter for crop growth monitoring, yield estimation and drought monitoring [2]. Large-scale soil moisture monitoring is an important

*e-mail: wang_jinliang@hotmail.com component of agricultural water management and crop drought monitoring [3]. The region of the Tropic of Cancer in Yunnan Province is characterized by unique geographical conditions, high vegetation coverage, outstanding biodiversity, abundant products (such as Panax notoginseng, purple rice, Punica granatum and Yunnan large leaf tea), and "living fossil" plants (such as Alsophila spinulosa and Manglietiastrum sinicum). The lack of soil moisture has a significant impact on the leaf area and yield of these crops [4]. The quality of crops can be improved and the efficiency of water resource utilization can be effectively increased by regulation 
of soil moisture. Therefore, monitoring of soil moisture content is of great significance and value for studying the impact of vegetation types.

Soil moisture monitoring methods include traditional methods and remote sensing methods. Traditional soil moisture monitoring methods, such as drying method, time domain reflectometer method, soil hygrometer method, neutron instrument measuring method, etc., although simple in operation and high in measurement accuracy $[5,6]$, are based on single-point monitoring, and it is difficult to realize real-time, large-scale, dynamic public opinion monitoring, which is not only time-consuming and labor-consuming, but also costly. Many researchers use soil moisture product data to verify and analyze soil moisture directly, and the results are highly correlated with the actual soil moisture [7, 8]. However, the spatial resolution of these data is less than $25 \times 25 \mathrm{~km}$, which cannot capture the changes of soil moisture at a fine scale and cannot meet the requirements of agricultural development and irrigation management [9]. Many researchers have performed research on soil moisture inversion using the remote sensing method and achieved good results [10-13]. The main methods include the thermal inertia method, evapotranspiration method, microwave remote sensing method, and vegetation index method [14]. The thermal inertia model is used to monitor soil moisture using thermal infrared remote sensing data [15]. Price [16] simplified the calculation of thermal inertia by summarizing the principle of thermal inertia remote sensing and proposed the viewpoint of apparent thermal inertia (ATI). The model is only related to solar radiation flux, surface albedo and diurnal temperature difference and is suitable for bare soil or sparse vegetation cover. The calculation for the evapotranspiration method requires numerous meteorological and surface parameters; thus, it is difficult to realize the process of remote sensing. The data for soil moisture monitoring by microwave remote sensing are difficult to obtain, and the cost is high. Based on monitoring of soil moisture by the vegetation index, many monitoring indicators have been established according to the characteristics of different regions, time and vegetation coverage $[11,17]$. The vegetation water supply index (VSWI) is suitable for seasons with strong vegetation transpiration, and the inversion accuracy is higher in areas with vegetation coverage greater than $30 \%$ [18, 19]. Because short-wave reflectivity (SWIR) is sensitive to leaf water content, the surface water capacity index (SWCI) of MODIS band 6 and band 7 can better reflect the surface water content anIn conclusion, due to the time-consuming and energy-consuming nature of traditional soil moisture monitoring, the low spatial resolution of soil moisture product data, and the difficulty of remote sensing monitoring methods, such as the evapotranspiration method, the microwave remote sensing monitoring process and related data acquisition, a lack of research still exists on soil moisture in the unique region of the Tropic of Cancer. Therefore, ATI, VSWI and SWCI are used to retrieve the soil moisture in the region of the Tropic of Cancer in Yunnan Province, and the optimal soil moisture retrieval method is selected by comparing and analyzing the soil volume moisture content. The retrieval model is validated by the temperature vegetation drought index (TVDI) [11], which can better reflect the soil moisture status and soil relative humidity (RM) [21] calculated by the empirical model of temperature difference vegetation thermal inertia. Finally, the soil moisture status is analyzed by combining the vegetation coverage and land use types.

\section{Materials and Methods}

\section{Study Area}

The study area is located in the counties (cities) crossed by the Tropic of Cancer in Yunnan Province $\left(23^{\circ} 26^{\prime} 21^{\prime \prime} \mathrm{N}\right)$, including Wenshan Prefecture, Honghe Prefecture, Yuxi City, Puer City, and Lincang Prefecture (5 prefectures/cities) and Funing, Xichou, Malipo, Yanshan, Wenshan, Mengzi, Gejiu, Shiping, Honghe, Jianshui, Yuanjiang, Mojiang, Ning'er, Jinggu, Shuangjiang, Cangyuan, and Gengma (17 counties/ cities), as shown in Fig. 1. The total area covers $57129.27 \mathrm{~km}^{2}$, the geographical position is located between $98^{\circ} 48^{\prime}-106^{\circ} 12^{\prime} \mathrm{E}$ and $22^{\circ} 02^{\prime}-24^{\circ} 10^{\prime} \mathrm{N}$, and the altitude is $107-3223 \mathrm{~m}$. The region is rich in light, hot water and gas, has high vegetation coverage, an excellent ecological environment, outstanding biodiversity, and is rich in characteristic products. The climate type is a subtropical monsoon climate with an average annual temperature of 15.9 to $24^{\circ} \mathrm{C}$ and an average annual precipitation of 770 to $2400 \mathrm{~mm}$. The dry and wet seasons are distinct. From May to October, this area is affected by the humid air flow, and more rainy days make up the rainy season. From November to April of the next year, the weather is sunny and dry under the influence of dry heating flow, which makes this area prone to drought and seriously affects agricultural production, quality of life and social economy. Therefore, taking January to May and November and December 2017 as the research period, soil moisture remote sensing monitoring and a timely understanding of the regional soil moisture information can supply technical support for water and soil regulation, which is of great significance to regional agricultural development.

\section{Materials}

\section{MODIS Data}

The remote sensing data used in the study were derived from the MODIS product data supplied by the National Aeronautics and Space Administration (NASA) official website, including the MOD09A1 surface reflectance $8 \mathrm{~d}$ synthetic product with a spatial resolution of $500 \mathrm{~m}$ and the MOD11A2 surface temperature $8 \mathrm{~d}$ synthetic product with a spatial resolution of 


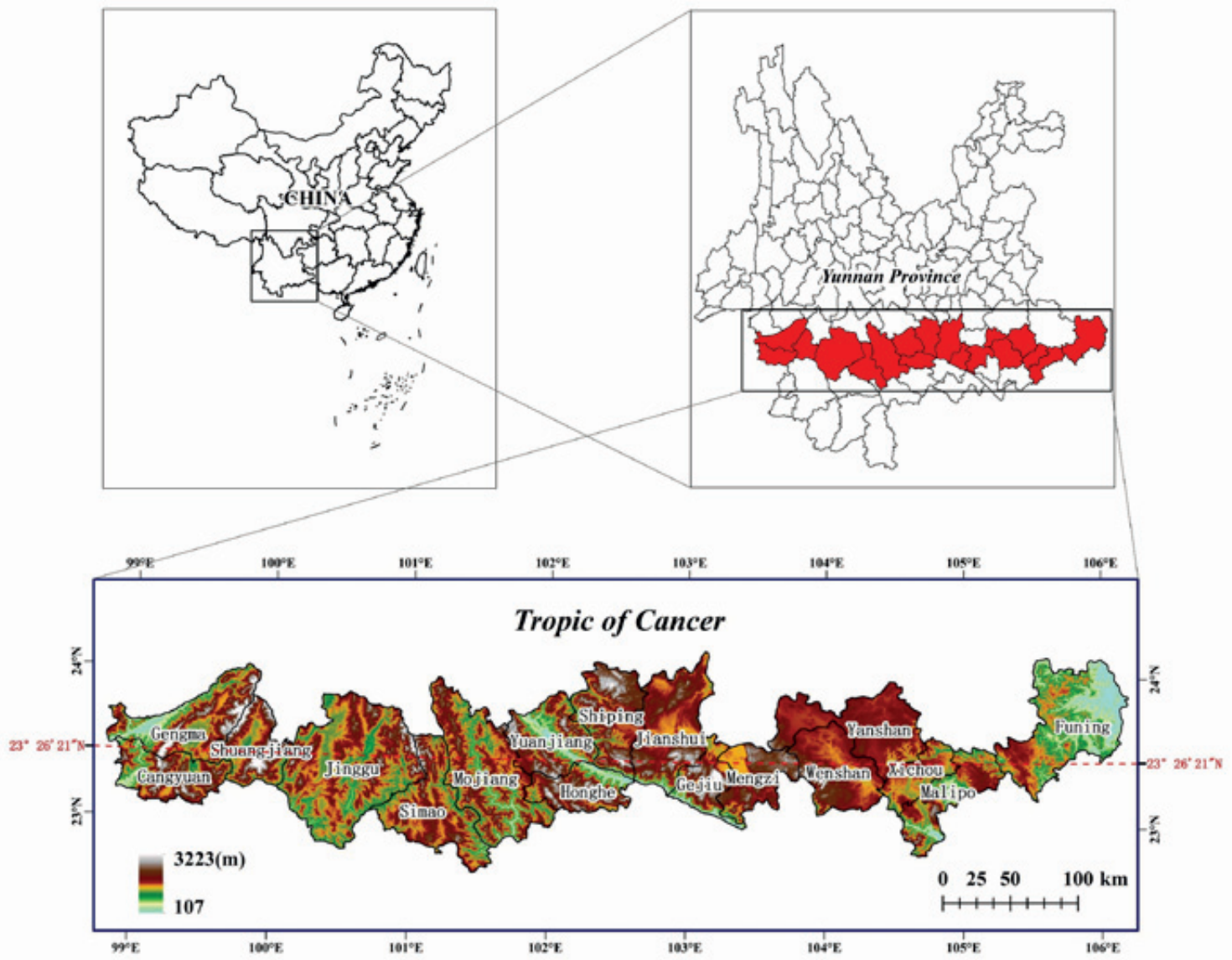

Fig. 1. Location map of the study area.

$1 \mathrm{~km}$. These data were obtained from January 2017 to December 2017. The MRT (MODIS Reprojection Tool) software developed by NASA was used to convert the MODIS data into a specific format. The data are resampled to $1 \mathrm{~km}$ via the cubic convolution method, which is consistent with the MOD11A2 resolution and the unified spatial coordinate system. Among these data, the MOD09A1 product data (actual resolution is $463.3 \mathrm{~m}$ ) of 1-7 band $8 \mathrm{~d}$ synthetic surface reflectivity use IDL to remove cloud and cloud shadow interference. The MOD11A2 product data (the actual resolution is $926.6 \mathrm{~m}$ ) include surface temperature during the daytime and night, emissivity in the 31 and 32 bands, and the day and night transit time, which effectively eliminates cloud interference.

\section{CLDAS-V2.0 Data}

The CLDAS atmospheric driving field products were issued by the National Meteorological Information Center of China. The selected ground observation data consist of the hourly temperature, air pressure, humidity, wind speed, precipitation and other data observed by more than 2,400 national automatic stations after quality control and nearly 40,000 regional automatic weather stations for business assessment. The system can supply a volumetric soil water content set $\left(\mathrm{m}^{3} / \mathrm{m}^{3}\right)$ of the $0-5,0-10,10-40,40-100$, and $100-200 \mathrm{~cm}$ vertical layers on an hourly or day-by-day basis at a spatial resolution of $0.0625^{\circ} \times 0.0625^{\circ}$. In the data product quality description, the CLDAS-V2.0 soil volumetric water content data product was evaluated using the soil moisture automatic station observation data after quality control in China's regional business. The results show that the CLDAS soil volumetric water content product has good agreement with the actual ground observation. The national average correlation coefficient is 0.89 , the root mean square error is $0.02 \mathrm{~m}^{3} / \mathrm{m}^{3}$, and the deviation is $0.01 \mathrm{~m}^{3} / \mathrm{m}^{3}$. Therefore, CLDAS-V2.0 can better reflect the spatial distribution of soil moisture, the local description is more accurate, and thus the product data can be used as a substitute for soil moisture. Hence, this study used the China Meteorological Administration's "Land Surface Data Assimilation System Real-Time Product Dataset (CLDAS-V2.0)" supplied by the China Meteorological Data Network to replace the measured data (http://data.cma.cn/). Mainly obtained from January 2017 to December 2017, the 0-10 cm daily soil moisture product data [22] uses the average of 8 days of monthly data as the monthly soil moisture data, consistent with the time resolution of the MODIS $8 \mathrm{~d}$ synthetic products. In this study, 80 CLDAS data samples were randomly selected to set up a regression equation for soil moisture retrieval by remote sensing. 


\section{Land Use Data}

The data were downloaded from the Resource and Environment Science Data Center of the Chinese Academy of Sciences based on Landsat 8 remote sensing images and generated by artificial visual interpretation. Land use types include the six primary types of cultivated land, forest land, grassland, water area, residential land and unused land and 25 secondary types. The data were acquired in 2015 with a spatial resolution of $1 \mathrm{~km}$.

\section{Methods}

The pre-processed MODIS data were used to calculate ATI, VSWI, and SWCI; the three indices were established with the soil moisture to establish a one-way regression model; and the optimal model was selected to invert the soil moisture. The vegetation coverage of the study area was extracted by the pixel binary model, and the soil moisture status of the study area was analyzed in combination with the land use type.

\section{Apparent Thermal Inertia (ATI)}

The thermal inertia is a thermal property of the soil and plays a role in the internal temperature change of the soil surface. Because of the high-water thermal conductivity and heat capacity, the soil thermal inertia is affected by the soil moisture, and the soil thermal inertia has a positive correlation with the soil moisture. The thermal inertia calculation formula requires additional physical parameters, and thus this study uses Price [16] to simplify the proposed ATI as one of the soil moisture inversion methods. The expression is given as follows:

$$
A T I=(1-A) / \Delta T
$$

In the formula, $A$ is the full-band albedo, $\Delta T$ is the difference of the surface temperature between day and night, and ATI is in the range of 0 to 1 . Liang came up with a general formula for MODIS data:

$$
\begin{gathered}
A=0.160 a_{1}+0.291 a_{2}+0.243 a_{3}+0.116 a_{4}+ \\
+0.112 a_{5}+0.081 a_{7}-0.0015
\end{gathered}
$$

...where $a_{1}-a_{5}$ and $a_{7}$ are the reflectivity of the corresponding bands of MODIS, which have been widely used by researchers [23].

\section{Vegetation Water Supply Index (VSWI)}

Under the same circumstances, if the soil water supply is sufficient, the plants display a good growth state, and plant transpiration is strong, resulting in a decrease in the canopy surface and soil temperature. When the vegetation is dry, to reduce water consumption, the vegetation pores are closed, transpiration is reduced, and the temperature on the canopy is increased. The canopy temperature of the crop can reflect the water supply status of the crop, and the linear slope of the canopy temperature with NDVI can reflect the regional soil moisture condition [24]. Therefore, the VSWI expression is obtained by combining the NDVI and canopy temperature:

$$
\begin{gathered}
V S W I=N D V I / T_{c} \\
\text { NDVI }=\left(\rho_{n}-\rho_{r}\right) /\left(\rho_{n}+\rho_{r}\right)
\end{gathered}
$$

...where NDVI is the normalized vegetation index and $T_{c}$ is the vegetation canopy temperature. When the vegetation coverage is high, the canopy structure is relatively uniform, and the difference between the surface temperature and vegetation canopy temperature is small if the study area belongs to a medium-high vegetation coverage area. Therefore, the surface temperature is used to replace the vegetation canopy temperature, and $p_{n}$ and $p_{r}$ are the surface reflectances of the near-infrared and infrared bands, respectively. The larger the VSWI, the stronger the transpiration of vegetation and the greater the soil water content, and vice versa.

\section{Surface Water Capacity Index (SWCI)}

Different soil types vary slightly with moisture, and generally decrease with the increase of soil moisture, which makes it possible to monitor soil moisture by remote sensing. By analyzing the water absorption curve [25] (Fig. 2), it is known that an absorption peak region occurs at the $1.45 \mu \mathrm{m}$ band and the $1.9 \mu \mathrm{m}$ band, and an absorption trough region is found at the $1.65 \mu \mathrm{m}$ band. In the vegetation spectrum reflectance curve, a reflectance trough appears at wavelengths of $1.45 \mu \mathrm{m}$ and $1.9 \mu \mathrm{m}$, respectively, corresponding to the absorption peak of the water reflectance curve, where the reflectance at the wavelength of $1.9 \mu \mathrm{m}$ is the most obvious. A peak of reflectance is observed at $1.65 \mu \mathrm{m}$, corresponding to the absorption trough of the water reflectance curve [26]. In the MODIS band, the corresponding peaks and troughs of the water reflection are found in band 6 $(1628-1652 \mathrm{~nm})$ and band $7(2105-2155 \mathrm{~nm})$, respectively. Both bands are located in the water vapor absorption region and are sensitive to the variation of water reflectivity. Considering the mixed difference between vegetation and soil, the index obtained by combining band 6 and band 7 of the MODIS data can reflect the surface water content to a certain extent. Du et al. [26] constructed the SWCI based on analysis of the characteristics of the water absorption curve and soil reflectance curve:

$$
S W C I=\left(b_{6}-b_{7}\right) /\left(b_{6}+b_{7}\right)
$$

...where $\mathrm{b}_{6}$ and $\mathrm{b}_{7}$ are the band 6 and band 7 reflectance values of MODIS data. The model has a positive 


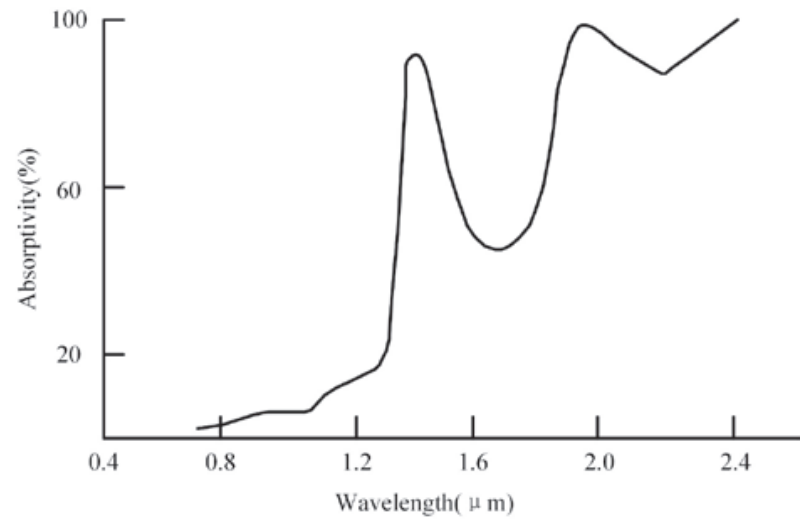

Fig. 2. Spectral absorptive curve of water.

correlation with water content and is less affected by vegetation cover.

\section{Fractional Vegetation Cover (FVC)}

Soil moisture is the key factor connecting climate change and vegetation cover dynamics, and soil moisture is the key factor connecting climate change and vegetation cover dynamics. The balance relationship of soil moisture is also different when vegetation cover is different. Therefore, it is necessary to analyze the relationship between vegetation cover and soil moisture. FVC is an index that reflects vegetation coverage evolved from vegetation normalization index, which can reflect the change of vegetation dynamically in time. The dimidiate pixel model is based on linear pixel decomposition method, which is relatively simple in form, which greatly weakens the influence of the atmosphere, soil background and vegetation type, and has good universality. The dimidiate pixel model considers a pixel to consist only of vegetation cover and

(a) Jan. 1

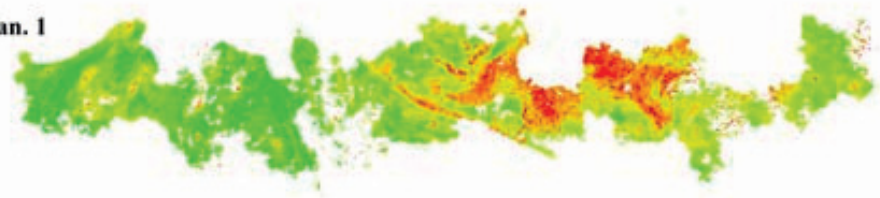

(b) Feb. 2

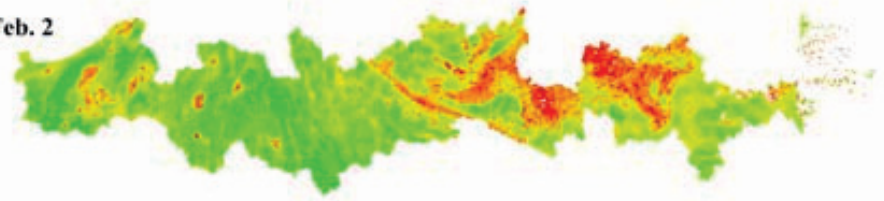

(c) Mar. 22

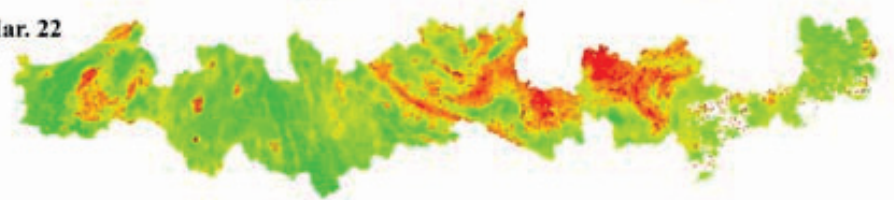

(d) Apr. 7

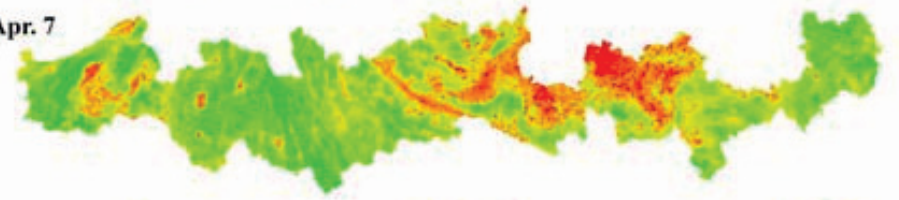

(e) May. 1

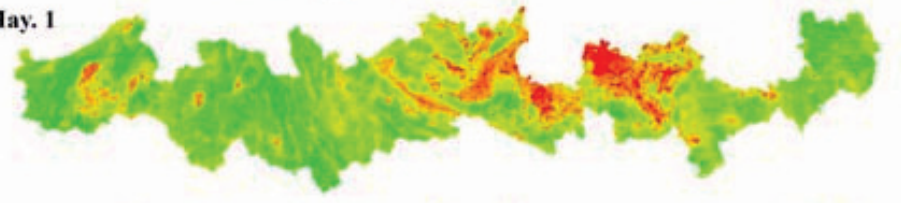

(f) Nov.1
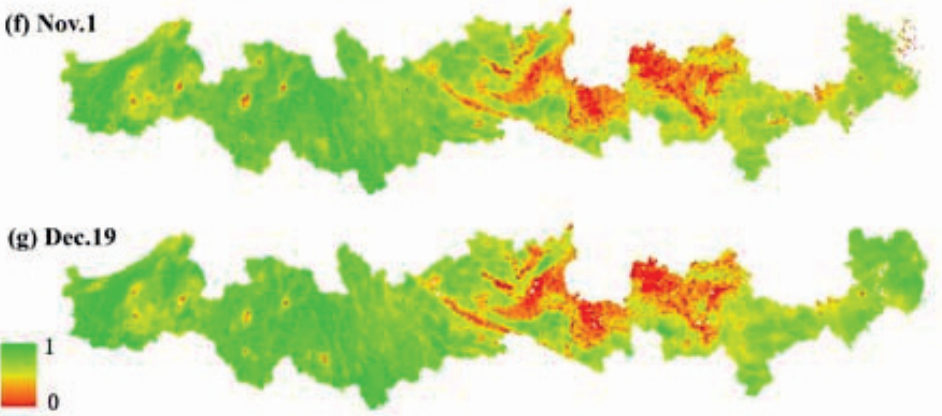

Fig. 3. FVC map. 
bare soil. The FVC pixel binary model formula is written as shown:

$F V C=\left(N D V I-N D V I_{\min }\right) /\left(N D V I_{\max }-N D V I_{\min }\right)$

...where $N D V I_{\max }$ and $N D V I_{\min }$ take on the maximum and minimum values within $1-99 \%$ of the confidence interval, and we divide the vegetation coverage into five grades (\%): 0-20, 20-40, 40-60, 60-80, and 80-100.

\section{Results and Discussion}

\section{Analysis of FVC}

Using the dimidiate pixel model to extract FVC from the Tropic of Cancer in Yunnan Province, the results show that except for Nov. 1, the trend of FVC in the other six periods is roughly the same. The FVC in Shiping County, Jianshui County, Gejiu City, Mengzi City, Yanshan County and Wenshan County areas is relatively low, and the FVC in the other areas is in the middle and high coverage area. The ATI value of the central region is low by the superposition of the ATI and the study area vector map, i.e., the ATI values of Shiping County, Jianshui County, Gejiu City, Mengzi City, Wenshan County and Yanshan County are low, and the ATI values of other areas are high, which is consistent with the spatial distribution of FVC. The distribution diagram of FVC is shown in Fig. 3.

\section{Analysis of Different Soil Moisture Characteristics}

\section{ATI Change Analysis}

According to formulas (1) and (2), ATI was calculated for each period from January 2017 to December 2017, and the spatial and temporal variations under different vegetation coverage levels were analyzed, as shown in Fig. 4. The results show that the distribution of ATI values in the study area in 2017 is $0.03-0.79$, mainly concentrated between 0.06 and 0.30 . The minimum, maximum and mean values are distributed between 0.03 and $0.07,0.17$ and 0.79 and 0.05 and 0.15 . With higher FVC level, the values of the minimum, maximum and mean values of the ATI value also increase, i.e., the larger the FVC, the larger the ATI value, and the ATI mean value in March-May 2017 is smaller than in other periods.

\section{VSWI Change Analysis}

The VSWI of each period from January 2017 to December 2017 was calculated according to formulas (3) and (4), and the water coverage pixels were removed. Combined with vegetation coverage, the distribution range of VSWI and its temporal and spatial variation under different grades of vegetation coverage were analyzed, as shown in Fig. 5. The results show that the VSWI value ranged from 0 to 0.57 , and the mean value was mainly between 0.06 and 0.10 . The greater the

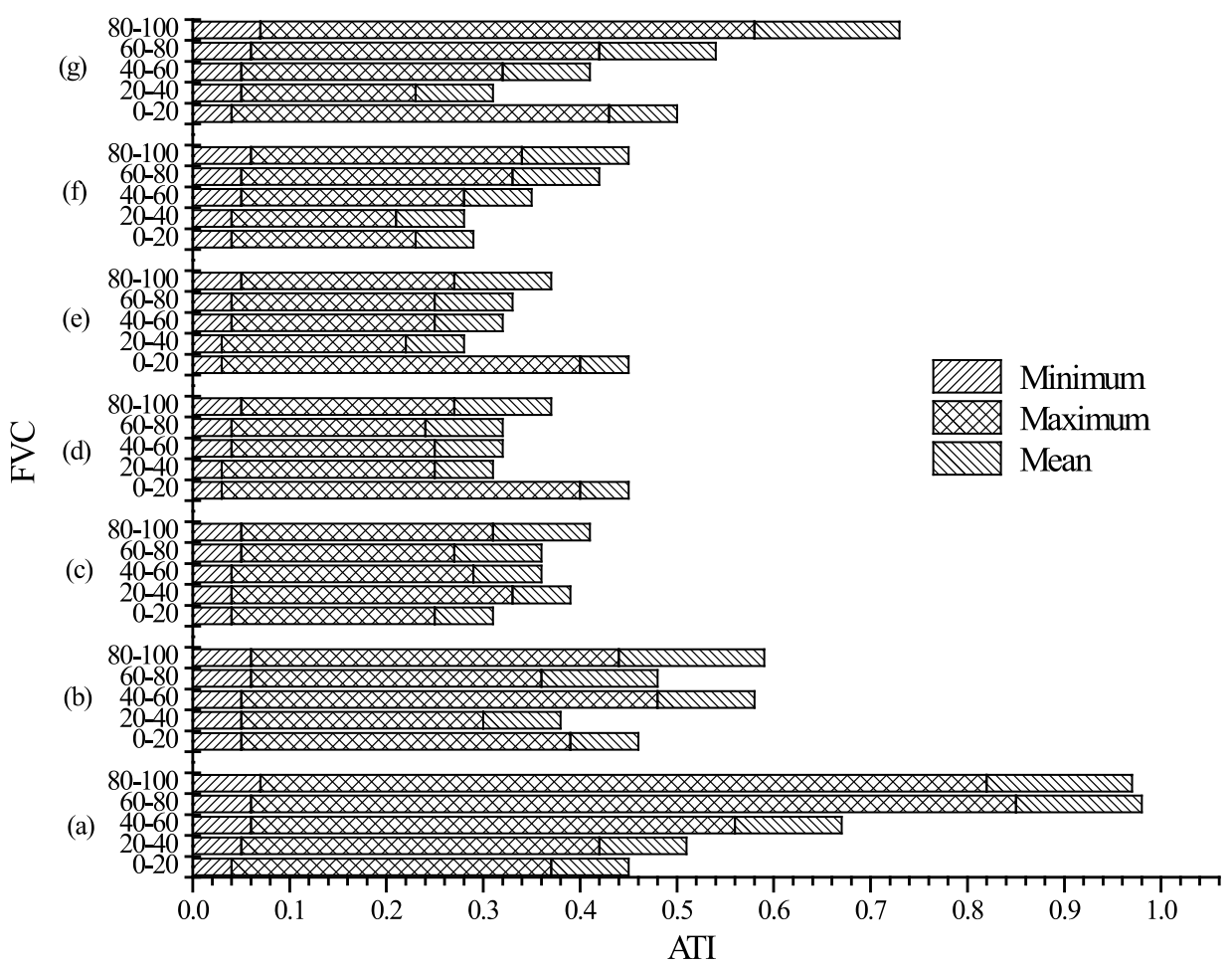

Fig. 4. ATI values under different FVC, where a), b), c), d), e), f), and g) are January, February, March, April, May, November, and December, respectively, and the same below. 


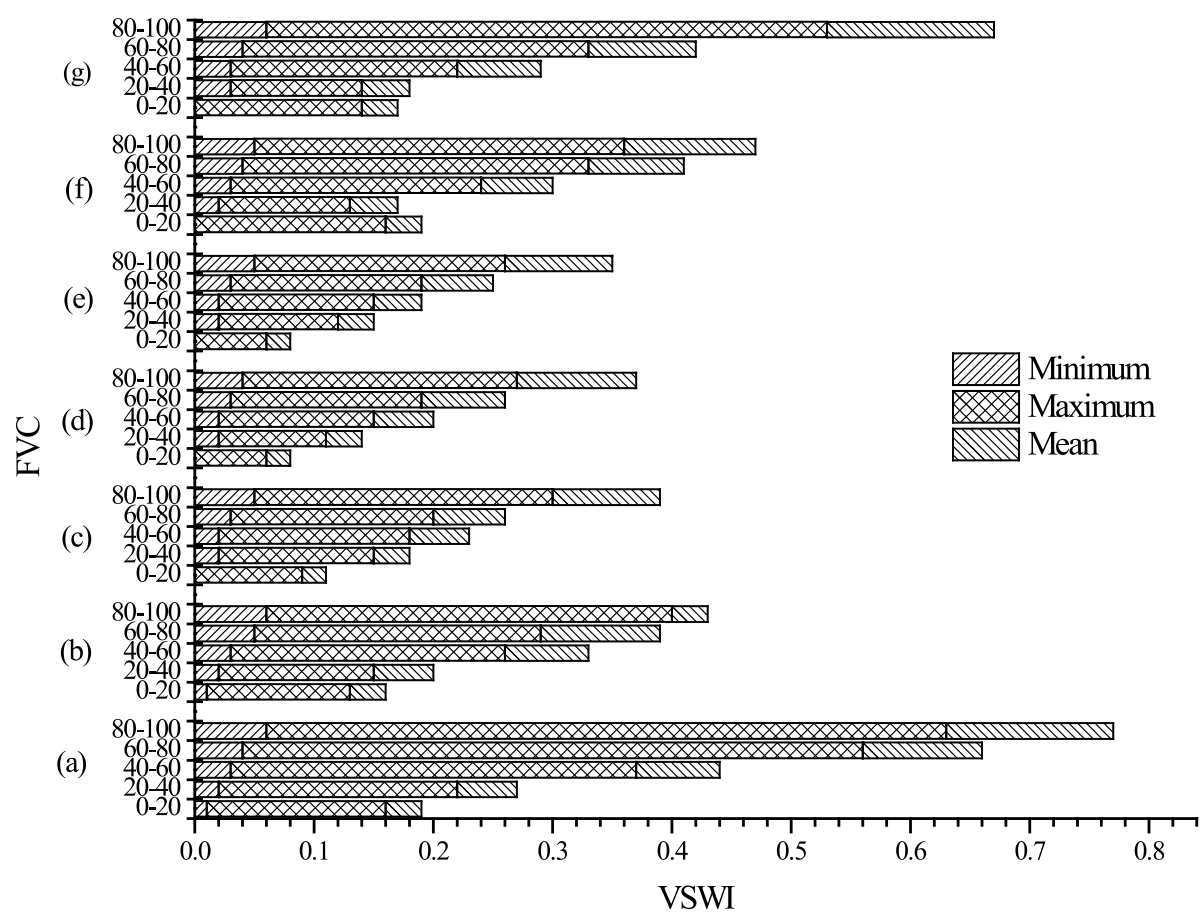

Fig. 5. VSWI values under different FVC.

vegetation coverage, the larger the VSWI value, and the spatial distribution of VSWI is consistent with FVC.

\section{SWCI Change Analysis}

Similarly, according to Formula (5) and FVC, by counting the SWCI values under different vegetation covers, as shown in Fig. 6, it is found that the SWCI range is $0.02-0.94$, and the mean value is between 0.13 and 0.49. As the SWCI level increases with the FVC level, its value also increases, and the larger the SWCI value, the more surface water content and the more humid the surface, and the index spatial distribution changes of FVC, ATI, and VSWI are consistent.

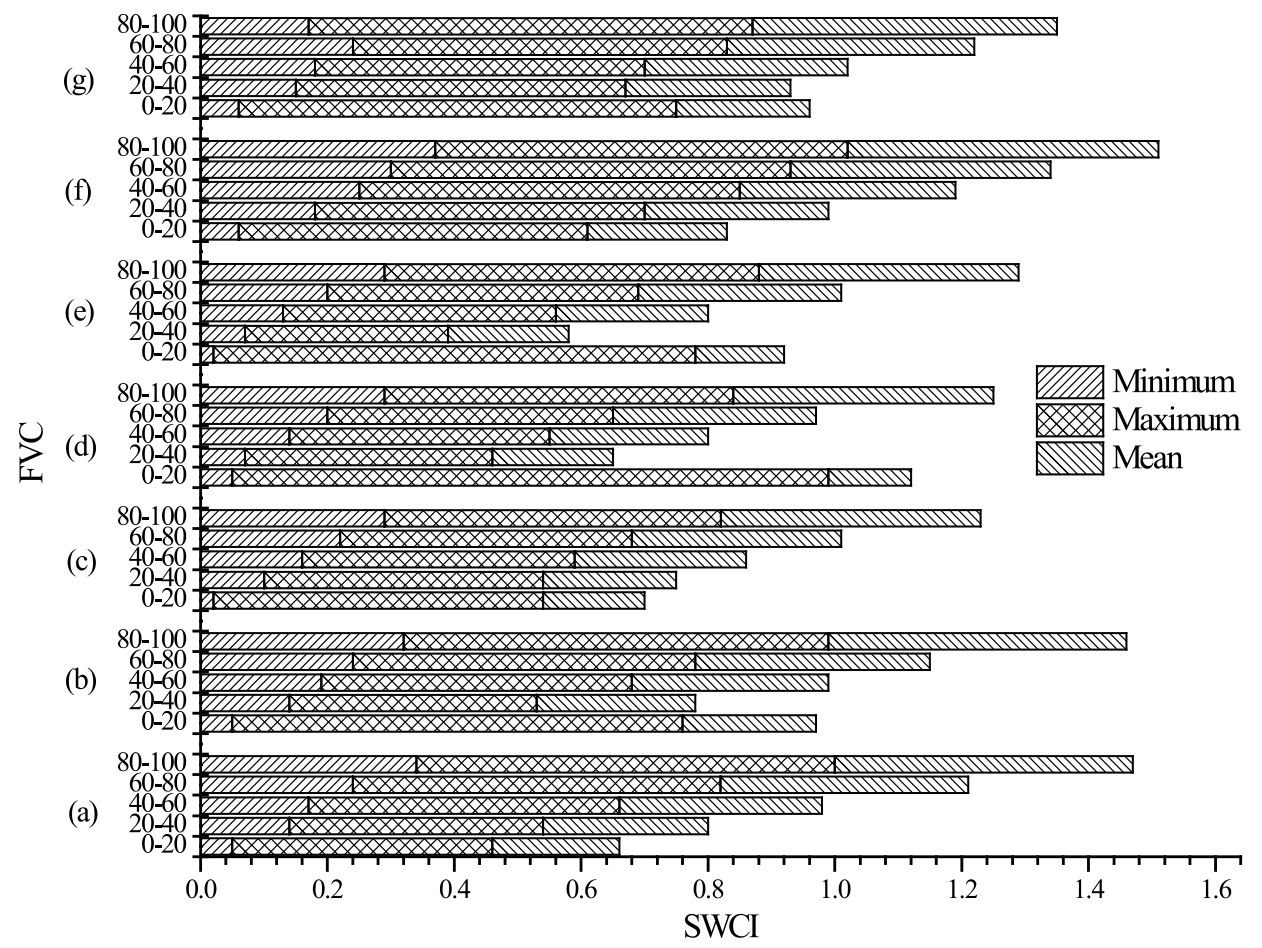

Fig. 6. SWCI values under different FVC. 
Table 1. Correlation between three indices and $\mathrm{SM}\left(\mathrm{R}^{2}\right)$

\begin{tabular}{|c|l|l|l|c|}
\hline Date & ATI & VSWI & SWCI & Samples \\
\hline Jan. 1 & $0.40^{* *}$ & $0.17^{* *}$ & $0.60^{* *}$ & 60 \\
\hline Feb. 2 & $0.66^{* *}$ & $0.72^{* *}$ & $0.62^{* *}$ & 70 \\
\hline Mar. 22 & $0.53^{* *}$ & $0.71^{* *}$ & $0.62^{* *}$ & 71 \\
\hline Apr. 7 & $0.58^{* *}$ & $0.69^{* *}$ & $0.62^{* *}$ & 71 \\
\hline May. 1 & $0.60^{* *}$ & $0.68^{* *}$ & $0.59^{* *}$ & 66 \\
\hline Nov. 1 & $0.59^{* *}$ & $0.64^{* *}$ & $0.54^{* *}$ & 68 \\
\hline Dec. 19 & $0.57^{* *}$ & $0.61^{* *}$ & $0.55^{* *}$ & 59 \\
\hline
\end{tabular}

Note: ${ }^{* *}$ means passes the reliability test of $\alpha=0.01$.

The above results show that the three indices are consistent with the distribution of vegetation cover. The higher the vegetation coverage grade, the greater the index value, and the three indices are positively correlated with soil moisture. Therefore, the soil water in Shiping County, Jianshui County, Gejiu City, Mengzi City, Wenshan County and Yanshan County is relatively low.

\section{Analysis of Characterization of Soil Moisture with Different Indices}

Using SPSS 22.0 software, the three indices of ATI, VSWI and SWCI were analyzed by linear regression analysis with the CLDAS soil moisture sample data to establish a regression equation. During the calculation using remote sensing data, the outliers affected by clouds (cloud shadows) and features were removed. Due to factors such as transient rainfall or artificial irrigation, soil moisture changes may not be continuous. Through the analysis of 80 CLDAS data samples, some certain extreme values and special values were excluded. The relevant analysis and test results are shown in Table 1.

Table 1 shows that the three indices have good correlation with soil moisture, and the coefficient of determination $\mathrm{R}^{2}$ is greater than 0.40 , indicating that all of the indices passed the reliability test of $99 \%$. Except for January, VSWI shows certain advantages in other months with $\mathrm{R}^{2}>0.61$. The soil moisture inversion effect was the best on February 2, which further indicates that VSWI can satisfactorily characterize the soil water capacity of the Tropic of Cancer in Yunnan Province. Except for January, March and April, the correlation between ATI and soil moisture is worse than that of SWCI, and the values for the other months are slightly larger than SWCI. Therefore, it can be observed that the two indices ATI and SWCI can better express the soil moisture inversion ability in this study area, and their $\mathrm{R}^{2}$ values are greater than 0.40 and 0.54 , respectively. Although SWCI also has a good correlation with soil moisture and the index is less affected by vegetation cover, the capability of the index is not as good as that of VSWI. Therefore, except in January, when the SWCI is selected to retrieve soil moisture, VSWI is used to invert soil moisture in the study area in other months. Fig. 7 shows the linear regression equation and scatter plot between the vegetation index with the highest $\mathrm{R}^{2}$ (i.e., optimal index) and the soil moisture in each month. Both VSWI and SWCI are positively correlated with soil moisture, $\mathrm{R}^{2}$ is greater than 0.59 , and the soil moisture is mostly greater than $0.2 \mathrm{~m}^{3} / \mathrm{m}^{3}$.
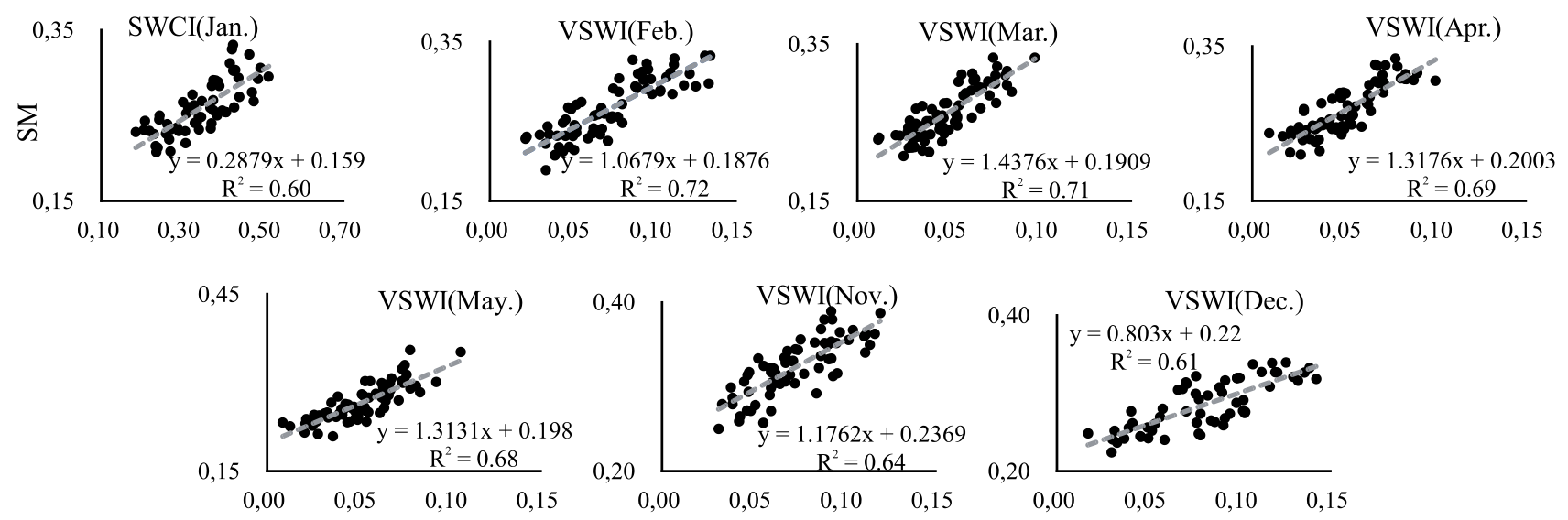

Fig. 7. Optimal index and SM simulation map (units: $\mathrm{m}^{3} / \mathrm{m}^{3}$ ). 
Table 2. Correlation between optimal index and TVDI, RM (R).

\begin{tabular}{|c|c|c|c|c|c|c|c|}
\hline Index & Jan. & Feb. & Mar. & Apr. & May. & Nov. & Dec. \\
\hline TVDI & -0.66 & -0.54 & -0.65 & -0.7 & -0.73 & -0.58 & -0.59 \\
\hline RM & 0.74 & 0.85 & 0.87 & 0.91 & 0.9 & 0.93 & 0.9 \\
\hline
\end{tabular}

To further illustrate the reliability of the retrieval model, TVDI and RM are used to analyze the correlation with the optimal index, as shown in Table 2. We found that the optimal index is negatively correlated with TVDI, and the absolute value of correlation coefficient $\mathrm{R}$ is greater than 0.54 , whereas the positive correlation with $\mathrm{RM}$ is greater than 0.74 .

Table 2 shows that the optimal index has a good correlation with TVDI and RM, and thus the model index for selecting the inversion is more reliable. Therefore, the seven regression equations in Fig. 7 are used to retrieve the soil moisture in each period, and the soil moisture retrieval map is obtained, as shown in Fig. 8.
The soil moisture content is divided into eight intervals $\left(\mathrm{m}^{3} / \mathrm{m}^{3}\right): \mathrm{SM}<0.1$ (I), $0.1 \leq \mathrm{SM}<0.15$ (II), $0.15 \leq \mathrm{SM}<0.20$ (III), $\quad 0.20 \leq \mathrm{SM}<0.25 \quad$ (IV), $\quad 0.25 \leq \mathrm{SM}<0.30 \quad$ (V), $0.30 \leq \mathrm{SM}<0.35 \quad$ (VI), $\quad 0.35 \leq \mathrm{SM}<0.40 \quad$ (VII) and $\mathrm{SM} \geq 0.40$ (VIII), and SM area in different intervals was calculated, as shown in Table 3.

Fig. 8 and Table 3 show that the soil moisture in the study area lies mainly between 0.2 and $0.4 \mathrm{~m}^{3} / \mathrm{m}^{3}$ (IV-VII). In terms of time distribution, the soil moisture content is the highest on November 1 compared with the remainder of the period followed by December 19 . The soil moisture content is the smallest on January 1 , and the soil moisture content is almost the same from

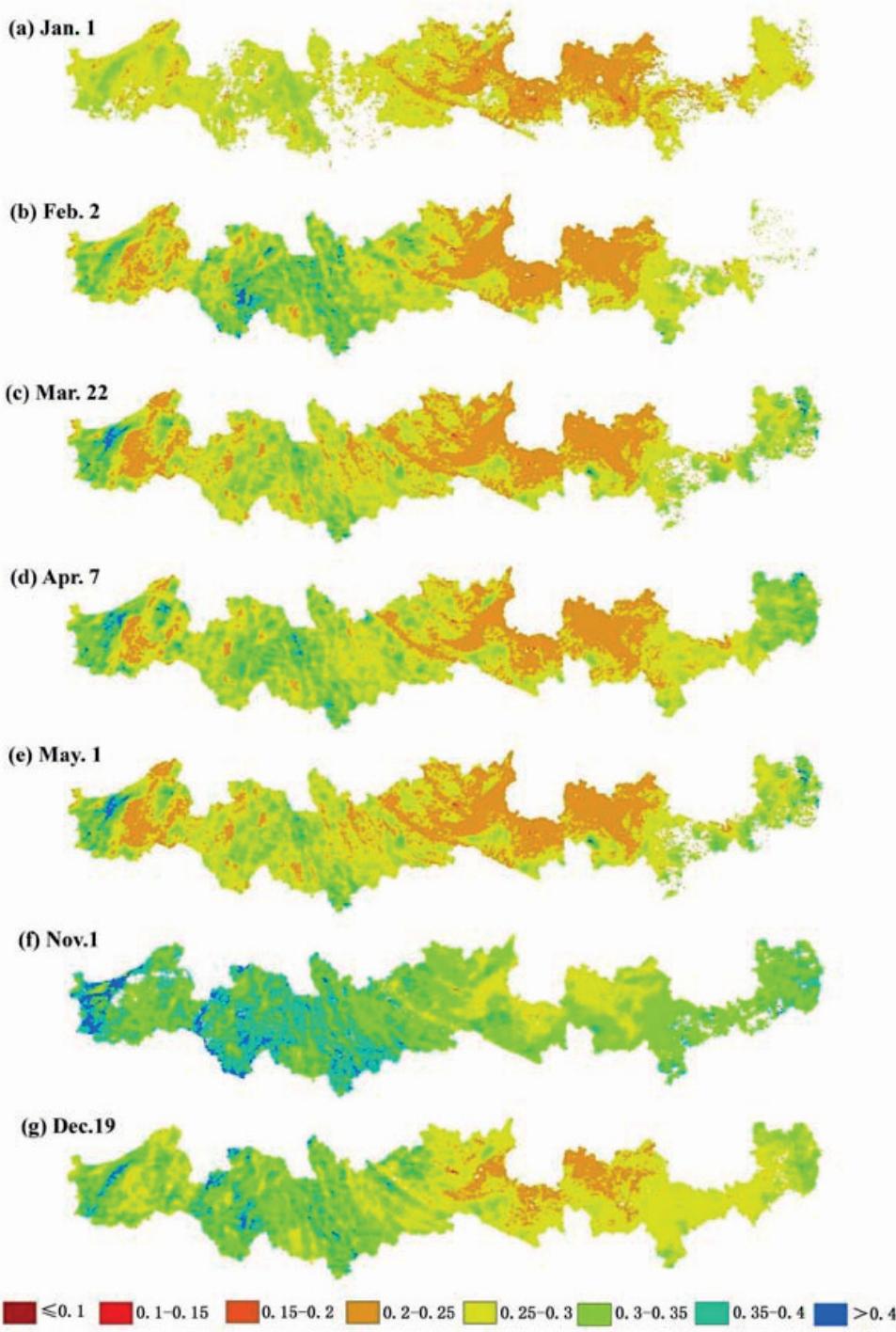

Fig. 8. SM distribution map in 2017 (units: $\mathrm{m}^{3} / \mathrm{m}^{3}$ ). 
Table 3. Statistics of the SM area of different regions in 2017.

\begin{tabular}{|c|c|c|c|c|c|c|c|c|}
\hline Date & I & II & III & IV & V & VI & VII & VIII \\
\hline Jan. 1 & 0 & 0 & 159 & 12171 & 28044 & 3793 & 1 & 0 \\
\hline Feb. 2 & 2 & 8 & 8 & 12671 & 22044 & 11989 & 2984 & 522 \\
\hline Mar. 22 & 2 & 6 & 15 & 15129 & 26054 & 9812 & 1358 & 342 \\
\hline Apr. 7 & 1 & 10 & 9 & 11529 & 26397 & 14197 & 2673 & 365 \\
\hline May. 1 & 0 & 6 & 10 & 11764 & 29229 & 12256 & 1654 & 99 \\
\hline Nov. 1 & 0 & 1 & 6 & 18 & 7614 & 28871 & 14130 & 2358 \\
\hline Dec. 19 & 1 & 8 & 8 & 4143 & 26406 & 19277 & 4326 & 640 \\
\hline
\end{tabular}

February to May. In terms of area distribution, on November 1 the soil moisture content is the largest in the range of $0.30-0.35 \mathrm{~m}^{3} / \mathrm{m}^{3}$ (VI) due to the rainy period from May to October, which is close to the rainy season, and the precipitation is greater, resulting in a larger soil moisture value in this period. The maximum area of soil moisture content in other periods is concentrated in the range of 0.25 to $0.30 \mathrm{~m}^{3} / \mathrm{m}^{3}(\mathrm{~V})$. In the $\mathrm{V}$ interval, the soil moisture area on May 1 reaches $29229 \mathrm{~km}^{2}$, which is due to the rainfall in most portions of the study area during this period. The area of soil moisture is the smallest in the range of $<0.20 \mathrm{~m}^{3} / \mathrm{m}^{3}$ (I-III). According to the literature data of the study area, drought occurs in spring and winter, the frequency of drought in Shiping County was higher than that in other counties, the drought season in Mengzi City was significant, and the drought in spring was more serious than that in autumn $[27,28]$. The rainfall in Wenshan Prefecture was the lowest from October to March of the following year, a period that was prone to drought [29]. The frequency of drought in Lincang station was higher in summer and winter [30], while in Yuanjiang County in Yuxi City, the temperature increased and the rainfall decreased [31].

\section{Soil Moisture Changes under Different Vegetation Covers}

Using the FVC (Fig. 3) and the type of land use, as shown in Fig. 9, the soil moisture under different vegetation coverages was analyzed, and the results show that the land cover type affects the soil water content. When the land use type is high-coverage grassland, medium-coverage grassland, forest land, shrub and open forest land, the soil moisture is almost always greater than $0.25 \mathrm{~m}^{3} / \mathrm{m}^{3}$. Soil moisture values less than $0.25 \mathrm{~m}^{3} / \mathrm{m}^{3}$ are mainly concentrated in land use types such as beach land, dry land, bare land and selected open forest land. This observation shows that the SWCI and VSWI indices selected for the study can better retrieve soil moisture.

With the support of ArcGIS technology, the partition statistics tool is used to obtain the mean value of the soil moisture at each level, as shown in Fig. 10. It can be observed that the change trend in the seven periods is the same, i.e., as the vegetation coverage increases, the soil water content also increases. In the seven periods, the average soil moisture value is greater than $0.2 \mathrm{~m}^{3} / \mathrm{m}^{3}$, and the average soil moisture values

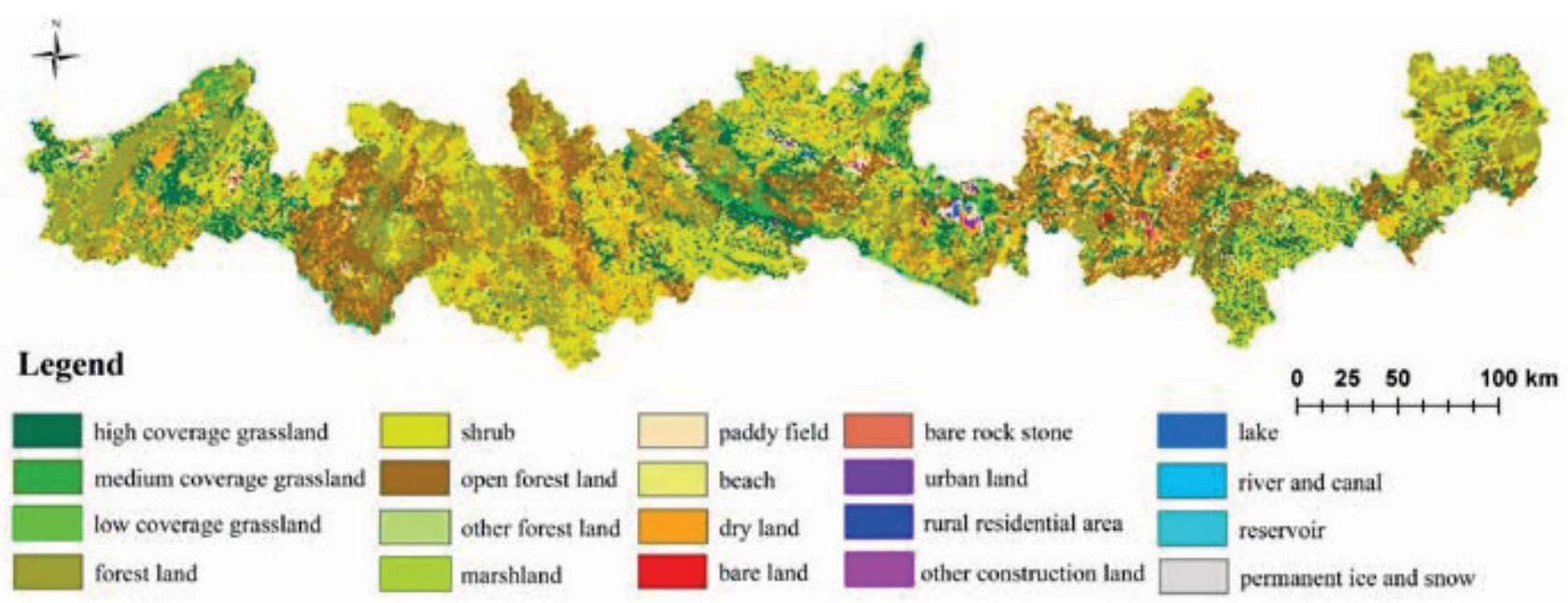

Fig. 9. Land use map in 2015. 


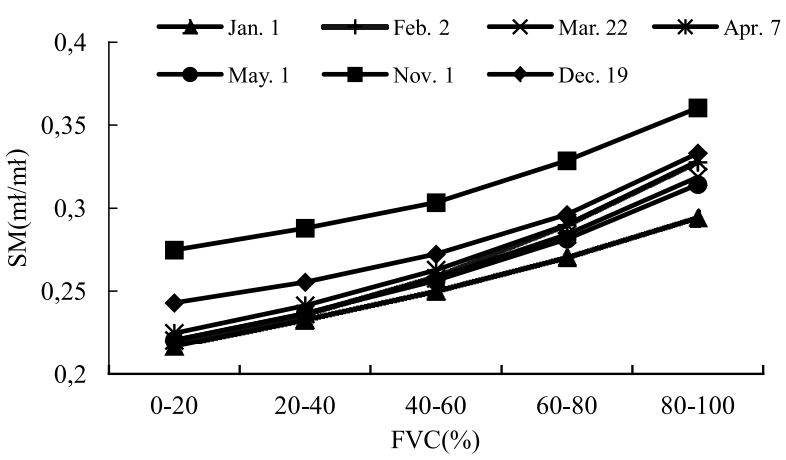

Fig. 10. Map of the mean value of SM at different FVC levels in different periods.

corresponding to different vegetation coverage levels are from Nov. 1 to Dec. 19, Apr. 7, Feb. 2, Mar. 22, May. 1, and Jan.1. According to statistics, FVC is greater than $40 \%$, accounting for approximately $81 \%$ of the study area, and thus VSWI has good retrieval ability in high and medium vegetation coverage areas.

\section{Discussion}

All of the soil moisture data retrieved by the three indices pass the reliability test of $99 \%$. ATI is suitable for low vegetation coverage [32], but according to the inversion results, ATI also has a certain indicative effect on soil moisture in the study area with high vegetation coverage, which is generally consistent with Song's view [23]. SWCI can satisfactorily reflect the surface water content and its change in the study area, which is consistent with Zhang's research results [33], and this index is suitable for remote sensing monitoring of shallow soil moisture. VSWI can satisfactorily reflect the soil moisture content in the Tropic of Cancer area of Yunnan Province with high vegetation coverage, which is consistent with Chen's view [34]. The retrieval results show that the soil water content is concentrated, and the smaller soil water content is concentrated in the central region. The study by Gao showed that the areas with the most serious drought conditions include Jianshui County, Yanshan County, and Wenshan County. This observation is due to the lack of surface water in the Karst area (such as Mengzi City and Jianshui County), the low-lying area, the valley area, and basin area, and with the alpine barriers around, less precipitation than around, coupled with large evaporation, makes conditions prone to drought [35].

\section{Conclusions}

Using MODIS product data (MOD09A1 and MOD11A2) as data sources, the ATI, VSWI, and SWCI indices were calculated. A single regression equation was constructed with the measured soil moisture, and a soil moisture inversion model suitable for the Tropic of Cancer in Yunnan was selected to produce the soil moisture distribution map. The FVC of the study area was extracted by the dimidiate pixel model, and the land use map was used to analyze the soil water content under different vegetation coverage and different land use types. We arrived at the following conclusions:

(1) The higher the FVC level, the larger the ATI, SWCI, and VSWI values, and the three indices show good correlation with soil moisture. In addition to January, VSWI has $\mathrm{R}^{2}$ greater than 0.61 in the remaining months, and the best fitting effect can be used to characterize soil moisture in the region. In the Tropic of Cancer in Yunnan Province, ATI and SWCI also have good correlation with soil moisture, and their $\mathrm{R}^{2}$ values are greater than 0.40 and 0.54 , respectively.

(2) The soil moisture in the study area is mainly concentrated between 0.2 and $0.4 \mathrm{~m}^{3} / \mathrm{m}^{3}$. In 2017, except for November 1, the soil moisture content is the largest. The soil moisture content is the smallest on January 1 , and the soil moisture changes during the remainder of the period are approximately the same. In Jianshui County, Mengzi City, Wenshan County, Yanshan County, Gejiu City and Shiping County, the soil moisture content is the smallest, and the large values of soil moisture are mainly distributed to the west of Gengma County, the west of Cangyuan County and the middle of Jinggu County. Except for January 1, the soil moisture in most areas is concentrated in the range of 0.25 to $0.30 \mathrm{~m}^{3} / \mathrm{m}^{3}$.

(3) The Tropic of Cancer area of Yunnan Province belongs to the middle and high vegetation cover area type, and the vegetation coverage in different periods is consistent with the change trend of soil moisture, i.e., with the increase in vegetation coverage, the soil water content gradually increases, and the average value of soil moisture is greater than $0.2 \mathrm{~m}^{3} / \mathrm{m}^{3}$. In high coverage grassland, medium coverage grassland, forest land, shrub land and open forest land, the soil moisture is almost always greater than $0.25 \mathrm{~m}^{3} / \mathrm{m}^{3}$, but the soil moisture values less than $0.25 \mathrm{~m}^{3} / \mathrm{m}^{3}$ are mainly concentrated in land use types, such as beach, dry land, bare land and portions of open forest land.

\section{Acknowledgments}

This work was supported by the Key Program of Basic Research of Yunnan Province of China for the "Scientific Investigation on the Geographical Environment of the Tourism Health Industry in the Tropic of Cancer (Yunnan Section) " [grant number 2019FA017], by the Graduate Research and Innovation Fundation Project of Yunnan Normal University of China [grant number yjs2018106], by the Erasmus+ Capacity Building in Higher Education of the Education, Audiovisual and Culture Executive Agency (EACEA) for the "Innovation on Remote Sensing Education and Learning"'grant number 586037-EPP1-2017-1-HU-EPPKA2-CBHE-JP], by Young and 
Middle-aged Academic and Technical Leaders Reserve Talents Training Program of Yunnan Province [grant number No.2008PY056], and by the Program for Innovative Research Team (in Science and Technology) in the University of Yunnan Province, IRTSTYN. Thanks to Yan Liu and Huan Deng of the Key Laboratory of Resources and Environmental Remote Sensing for Universities in Yunnan for their help in experimental processing and analysis.

\section{Conflicts of Interest}

The authors declare no conflicts of interest.

\section{References}

1. ZHANG X., CHEN B.Z., ZHAO H., LI T., CHEN Q.F. Physical-based soil moisture retrieval method over bare agricultural areas by means of multi-sensor SAR data. International Journal of Remote Sensing, 39, 3870, 2018.

2. ZHANG X., CHEN B.Z., ZHAO H., WANG L. Soil moisture change detection over bare agricultural area by means of time-series Sentinel-1A SAR data. Remote Sensing Technology and Application, 32 (2), 338, 2017.

3. CHEN T., ZHUO,G., BA L.A. Study on soil moisture by remote sensing retrieval based on MODIS data in Eastern Naqu. Chinese Journal of Soil Science, 48 (2), 298, 2017.

4. WANG J.C., XU C.L., HAN X.F., GAO S., XU Y.L. Effects of different soil moisture on growth,grain yield and water use efficiency of spring wheat under drip irrigation. Research of Agricultural Modernization, 32 (1), 115, 2011.

5. CHU D. MODIS remote sensing approaches to monitoring soil moisture in Tibet, China. Remote Sensing Letters, 9 (12), 1148, 2018.

6. HOU L.Q., ZHANG J. Spatiotemporal variation of surface soil moisture based on temperature vegetation dryness index (TVDI) in Qinshui coalfield. Research of Soil and Water Conservation, 24 (3), 177, 2017.

7. HAN S., SHI C.X., LIN H.J., MENG X.Y., LV H.Q. The CLDAS soil moisture operation products applied to monitor soil drought. Journal of Glaciology \& Geocryology, 37 (2), 446, 2015.

8. XI J.J., WEN J., TIAN H., ZHANG T.T. Applicability evaluation of AMSR-E remote sensing soil moisture products in Qinghai-Tibet plateau. Transactions of the Chinese Society of Agricultural Engineering, 30 (13), 194,2014.

9. FAN L. Multi-source data estimating soil moisture and its application on forest fire risk assessment. The University of Chinese Academy of Sciences, 2017.

10. PETROPOULOS G.P., IRELAND G., BARRETT B. Surface soil moisture retrievals from remote sensing: Current status, products \& future trends. Physics and Chemistry of the Earth, Parts A/B/C, 83, 36, 2015.

11. CHEN C.F., SON N.T., CHEN C.R., CHIANG S.H., CHANG L.Y., VALDEZ M. Drought monitoring in cultivated areas of Central America using multi-temporal MODIS data. Geomatics, Natural Hazards and Risk, 8 (2), 402, 2017.

12. NAM W.H., TADESSE T., WARDLOW B.D., HAYES M.J., SVOBODA M.D., HONG E.M., PACHEPSKY Y.A.,
JANG M.W. Developing the vegetation drought response index for South Korea (VegDRI-SKorea) to assess the vegetation condition during drought events. International Journal of Remote Sensing, 39 (5), 1548, 2018.

13. BUSHRA N., ROHLI R.V., LAM N.S., ZOU L., MOSTAFIZ R.B., MIHUNOV V. The relationship between the normalized difference vegetation index and drought indices in the South Central United States. Natural Hazards, 1, 2019.

14. ZHANG Q.R. Study of drought monitoring by remote sensing based on MODIS data in Henan Province. Nanjing University of Information Science \& Technology, 2015.

15. MATSUSHIMA D., ASANUMA J., KAIHOTSU I. Thermal inertia approach using a Heat budget model to estimate the spatial distribution of surface soil moisture over a semiarid grassland in Central Mongolia. Journal of Hydrometeorology, 19 (1), 245, 2018.

16. PRICE J.C. The potential of remotely sensed thermal infrared data to infer surface soil moisture and evaporation. Water Resources Research, 16 (4), 787, 1980.

17. PEI F.S., WU C.J., LIU X.P., LI X., YANG K.Q., ZHOU Y., WANG K., XU L., XIA G.R. Monitoring the vegetation activity in China using vegetation health indices. Agricultural and Forest Meteorology, 248, 215, 2018.

18. SONG Y., FANG S.B., YANG Z.Q., SHEN S.H. Drought indices based on MODIS data compared over a maizegrowing season in Songliao Plain, China. Journal of Applied Remote Sensing, 12 (4), 046003, 2018.

19. CUNHA A., ALVALA R.C., NOBRE C.A. CARVALHO M.A. Monitoring vegetative drought dynamics in the Brazilian semiarid region. Agricultural and forest meteorology, 214, 494, 2015.

20. ZHANG H.W., CHEN H.L. The Analysis and Application of Soil Texture on Soil Moisture Retrieval. Chinese Journal of Soil Science, 44 (5) , 1086, 2013.

21. LI F.H. Study and Application for Soil Moisture Monitor Using Remote Sensor Technology in the Semiarid Area of Heilongjiang Province. Jilin University, 2006.

22. YAN H., ZHOU G., LU X. Comparative analysis of surface soil moisture retrieval using VSWI and TVDI in karst areas. In Proceedings of International Conference on Intelligent Earth Observing and Applications, 2015.

23. SONG Y., FANG S.B., LIANG H.Y., KE L.n. Comparison and application of agricultural drought indexes based on MODIS data. Remote Sensing for Land \& Resources, 29 (2), 215, 2017.

24. TRISASONGKO B.H., PANUJU D.R., SHIDDIQ D., LA ODE S.I., SHOLIHAH R.I., KUSDARYANTO S. Constraints of VSWI in the estimation of drought extent using Landsat data: A case of Tuban, Indonesia. Procedia Environmental Sciences, 24, 25,2015.

25. ZHANG H., CHEN H., SUN R., YU W., ZOU C., SHEN S. The application of unified surface water capacity method in drought remote sensing monitoring. Proceedings of SPIE - The International Society for Optical Engineering, 7472, M-1, 2009.

26. DU X., WANG S.X., ZHOU Y., WEI H. Construction and validation of a new model for unified surface water capacity based on MODIS data. Geomatics and Informa tion Science of Wuhan University, 32 (3), 205, 2007.

27. HAN Q.L., TANG H.P. Analysis on the characteristics of meteorological drought and climate in Honghe Prefecture Yunnan Province in recent 50 years. Journal of Guizhou Meteorology, 38 (5), 17, 2014. 
28. WANG F.F., WANG Q.F., LI R. Analysis on the causes and prevention of spring drought in Mengzi City, Yunnan Province. Education of Geography, 4, 51, 2016.

29. WANG L., LONG C.H., ZHENG F. Analysis on climate characteristic of severe drought in Wenshan Prefecture. Yunnan Geographic Environment Research, 23 (supp.), 14, 2011.

30. HAN Y.Y., LIU H. Study on evaluation of drought in central north region of Yunnan Province from 1954 to 2012. Journal of Water Resources \& Water Engineering, 26 (1), 111, 2015.

31. LIU Y.T., JIN Y.Q., LI J., ZHANG Y.P., LI P.G. Variation characteristic of soil moisture and soil temperature in Yuanjiang savanna ecosystem and its response to drought. Mountain Research, 36 (6), 790, 2017.
32. WU J.W., SUN L.Y., ZHANG Y.S., RUI F., JI R.P., ZHANG S.J., YU W.Y. Comparative study on the methods of estimating soil moisture by remote sensing under different vegetation cover. Chinese Agricultural Science Bulletin, 30 (23), 303, 2014.

33. ZHANG H.W., CHEN H.L., SHEN G.H., YU W.D. Drought remote sensing monitoring based on the surface water content index(SWCI) method. Remote Sensing Technology \& Application, 23 (6), 624, 2008.

34. CHEN S.D. Research on remote sensing inversion of soil moisture in Zhaokou irrigation of Henan. Zhengzhou University, 2014.

35. HAN Y., JIANG X.J. Analysis on drought characteristics in Yunnan Province and determination of typical drought year. Water Resources Development and Management, 2, $52,2018$. 
